\title{
A Study on the effect of stroop test on the formation of students discipline by using the heart rate variability (HRV) technique
}

\author{
Shahidah Hamzah ${ }^{1 *}$, Fauziah Ani ${ }^{2}$, Harliana Halim ${ }^{3}$, Shamsaadal Sholeh Saad ${ }^{4}$, Siti Sarawati Johar ${ }^{5}$, Zahrul \\ Akmal Damin ${ }^{6}$, Lutfan Jaes ${ }^{7}$, Muhammad Nubli Abdul Wahab \\ ${ }^{1,2,3,4,5,6,7}$ Pusat Pengajian Umum dan Ko Kurikulum, Universiti Tun Hussien Onn, Batu Pahat, Johor, Malaysia \\ ${ }^{8}$ Pusat Bahasa Moden dan Sains Kemanusiaan, Universiti Malaysia Pahang, Gambang, Kuantan, Malaysia \\ *Corresponding author E-mail: shahidahh@uthm.edu.my
}

\begin{abstract}
Discipline refers to self-control and individual behaviour. Other than that, discipline is an important element in the formation of integrity level. The objective of the study is to assess the effects of using the Stroop test of biofeedback protocol in order to evaluate individual level of discipline. A clinical study has been conducted on 50 participants which is the participants is a undergraduate student from Universiti Malaysia Pahang, who were divided into two groups. First group is students get high achiever and second group is students get low achierver in academic. The Heart Rate Variability (HRV) technique has been used in the assessment of this protocol. The findings show that there was a positive relationship between the Stroop test and the students discipline that those who excelled managed to get higher score of LF spectrum as compared to HF and VLF, while the students with lower achievement showed higher score of VLF and HF spectrum than LF. In conclusion, this test is one of the tests that can be used in increasing the level of individual discipline.
\end{abstract}

Keywords: Stroop Test; Discipline; Heart Rate Variability

\section{Background}

According to Foucault (1), discipline is a form of control and getting used to certain desired practices. As stated by Cawood (2), in general, psychology plays an important role in influencing the formation of discipline and will affect individual achievement. In explaining the relationship between discipline and psychology, the structures and organ systems of the human body play an indirect role (3).

Autonomic nervous system ANS is divided into two parts, namely the sympathetic nervous system (SNS) and parasympathetic nervous system (PNS) (4). According to Ellenby et al. (5), SNS plays the role of fight or flight in the nervous system while PNS functions to control and calm down. The balance between SNS and PNS is referred to as homeostasis. This balance will regulate the formation of individual behaviour in solving the conflict within themselves.

According to Akselrod et. al. (6) the activation of SNS and PNS will have an impact on whether the human body is stressed out or relaxed. The balance between ANS subsystems is important in maintaining homeostasis and thus more positive action can be taken. Heart Rate Variability (HRV) is a cardiovascular signal generated through blood pressure (BP) in the human body system. According to McCraty et. Al. (7) and Wijsman et al. (8), by means of HRV, the level of mental health and ANS can be assessed through the score values of HRV spectrum. The HRV spectrum score is a measure of the change in heart rate caused by physiological phenomenon. There are three power spectrum adopted; the VLF $(0-0.94 \mathrm{~Hz})$, HF $(0.15-0.4 \mathrm{~Hz})$ and LF $(0.04-0.15 \mathrm{~Hz})(9)$. As stated by Kamath \& Fallen (10), the frequency produced reflects the SNS and PNS activities which are involved in ANS.
The HF power spectrum is associated with PNS that involves respiratory sinus arrhythmia (RSA) which is an increase of HR during inspiration and decrease of HR during expiration. The LF power spectrum is a combination of SNS and PNS modulations, while VLF power spectrum represents the vasomotor system. The score of HRV spectrum will increase the score of HF and VLF spectrums in a stressed individual while when the individual is in a state of rest emotionally or physically, the score of LF spectrum will increase (11). The results of a study carried out by Akselrod et. al. (6) find that when an individual is in a high level of stress and out of control emotions, ANS will react in which SNS is activated. This is due to the increase in BP and muscles and then a change in the score of HRV spectrum takes place.

In assessing the relationship between discipline and a group of students who achieved high or low academic achievement, stroop test has been used. The findings of a study conducted by Lohr (12) show that the Stroop test is a method that can be used in the diagnosis of depression levels in an individual compared to the normal controlled one. These findings are supported by a study conducted by Imbrosciono and Berlach (13), which states that the Stroop test is a strong predictive tool in assessing the levels of individual performance and behaviour. Therefore, this study will emphasize on the effects of the Stroop test on the formation of students discipline by using HRV method.

\section{Methodology}

The research methodology discussed in this study includes the participants, apparatus, procedure and data analysis. The objective of the study is to evaluate the effects of Stroop test on the formation of the students discipline by using the HRV method. The main biofeedback equipment being used in the experiment was 
Biograph Infinity Software in order to detect their emotions through their heart rate rhythm.

\subsection{Participants}

To achieve the objectives of the study, 50 participants had been selected through systematic random method that involved students from Universiti Malaysia Pahang and Universiti Tun Hussien Onn Malaysia. The study participants were divided into two groups; a group of those who obtained excellent CGPA (3.49 - 4.00) and a group of those who received passing status (2.00 - 2.49).

\subsection{Apparatus}

In order to achieve the purpose of the study, the main instruments used were Pro Comp 2 and BVP sensor. These instruments are developed by Thought Technology Ltd. (Figure 1 and 2). Pro Comp 2 is a system that has been existed in conducting study about biofeedback. With the aid of this instrument, human physiological unit can be measured consisting of EMG, GSR, HRV and Resp. The data obtained from this method will be recorded in the Multimedia Biofeedback Biograph Infiniti Software (Figure 3), and then will be analysed. In addition, the BVP sensor will act to detect heartbeats based on photopletysmography principle which involves measuring the amount of infrared light absorbed by blood and in turn will cause changes to the cardiac cycle channel (14, 15). A BVP sensor will be placed on the non-dominant finger. Movements will affect the signal and will create atrifak on data to be recorded. The unit used for measuring BVP is beats per minute (bpm) and parameters that can be viewed using this device includes HRV spectrum and relative blood flow.

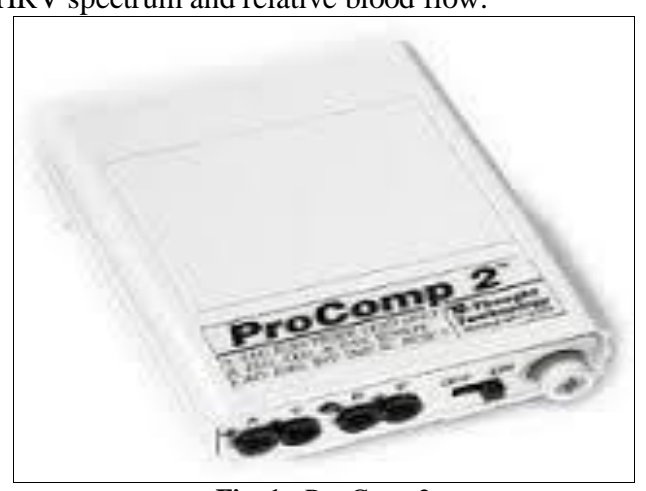

Fig. 1: Pro Comp2

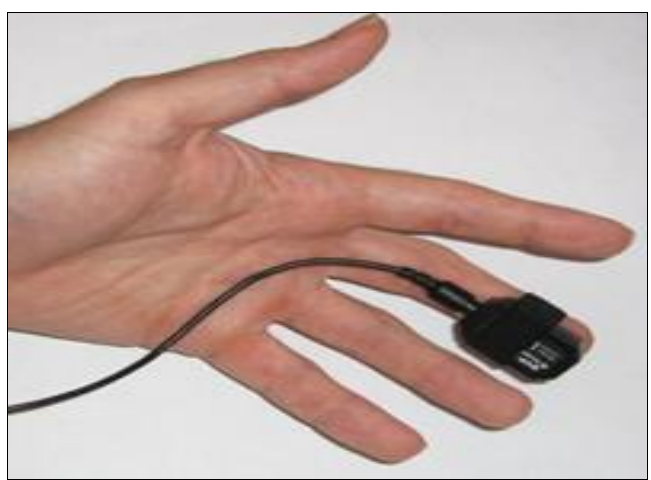

Fig. 2: BVP Sensor

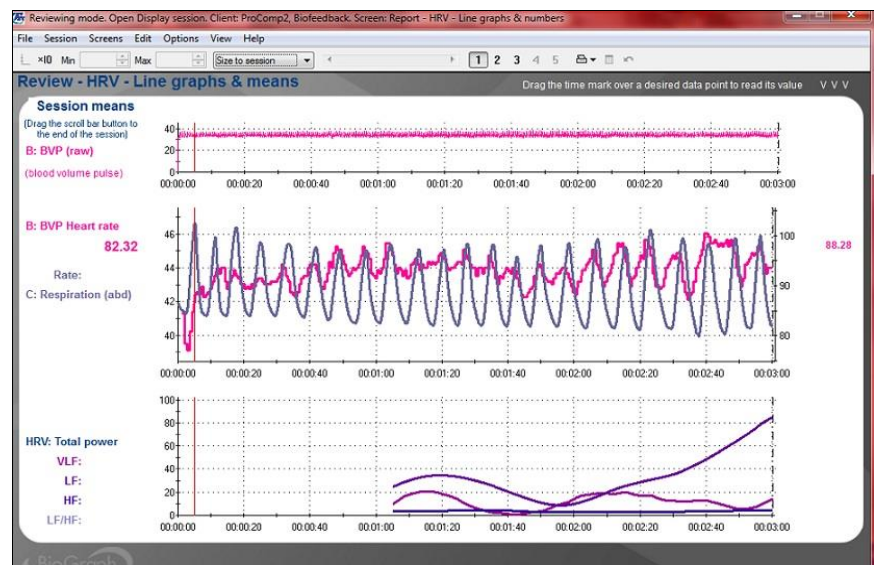

Fig. 3: Example of HRV Spectrum's Score Data that have been recorded via Multimedia Biofeedback Software

Source: Thought Technology Ltd.

\subsection{Procedure}

In this study, participants were divided into two groups; group A for the participants who obtained excellent academic achievement and group B for the participants who obtained passing status academic achievement. Each participant will undergo the Stroop test, which emphasizes on the use of time and good listening-tocommand skills. According to Siska (16), the effect of performance in the stroop test can determine the relationship between observation and physiological activity is in tandem with which to evaluate the level of performance of individual achievement in carrying out a case. Individuals with high level of effort will be able to balance their recovery level after the test is given.

Apart from that, this test also aims to assess individual concentration levels as through these activities, conflicting thoughts will occur. This Stroop test will take approximately 2 minutes for each participant. During this period, participants must complete two sections of tasks; in section A participants must state the colour they see of the words provided from top to bottom while in section B participants must state the colour they see of the words provided from bottom to top.

Table 1: Section A

\begin{tabular}{|l|l|l|l|l|}
\hline Red & Green & Pink & Grey & Black \\
\hline Pink & Red & Black & Green & Red \\
\hline Green & Black & Green & Red & Pink \\
\hline Grey & Pink & Red & Black & Green \\
\hline Black & Grey & Grey & Pink & Grey \\
\hline
\end{tabular}

Table 2: Section B

\begin{tabular}{|l|l|l|l|l|}
\hline Red & Red & Blue & Red & Gray \\
\hline Gray & Red & Pink & Pink & Blue \\
\hline Pink & Blue & Red & Gray & Red \\
\hline Red & Gray & Red & Red & Pink \\
\hline Blue & Pink & Gray & Blue & Red \\
\hline
\end{tabular}

During this test, the instructor will record the data in terms of time and error that was made. In addition, the participant's heart rate will be measured using the pro comp 2 through BVP sensor. The data will be recorded via Multimedia Biofeedback Software to detect the emotional levels of the participants based on the pattern of the heart rate rhythm. The scores of HRV spectrum will be divided into three, namely, Very Low Frequency (VLF) where the score range is between 0.0033 and $0.04 \mathrm{~Hz}$, Low Frequency (LF) refers to the score of spectrum between 0.04 and $0.15 \mathrm{~Hz}$ and $\mathrm{High}$ Frequency (HF), the score of spectrum between 0.15 and $0.4 \mathrm{~Hz}$ (Clinical Manual Ver 3.0). The recorded data will be analysed and the discipline levels of the participants can be assessed.

\section{Results and Discussions}

This section will discuss the impact of Stroop test on the formation of participants' discipline elements. Other than that, the 
data recorded by the instructor of the time and errors made will also be described in more detail.

\subsection{HRV Performance of the Participants}

The findings of the study show that there are differences in patterns of the heart rhythm for participants from group A and group B. Based on Figure 1, group A participants scored high percentage of LF of 41.97 percent compared to VLF (16.55\%) and HF $(41.4 \%)$. However, the difference in the score percentage between LF and HF is only 0.48 percent. This shows that the participants of group A were able to control themselves and were in a calm state throughout the test. Therefore, the group A participants have the ability to control the breathing pattern which resulted in slow heart rate, thus increased their HRV score.

A study by Cahill \& Alkire (17) and Shah et al. (18) states that the effect of SNS in improving the memory of individuals finds that the activation of SNS will increase individuals effort and further improve their performance in terms of a better time management and more focused when dealing with conflicts. In addition, Frewen et al. (19) report that the higher the score of LF spectrum, the better the individual cognitive assessment and performance, and in turn will improve the discipline and sense of responsibility of the individual.

The group B participants on the other hand, obtained a higher percentage of VLF score (44.29\%) than HF (36.90\%) and LF $(19.42 \%)$. The percentage difference between VLF and LF is $24.87 \%$. This shows that there is a large difference in the range between VLF and LF scores. This shows that the participants of group B have failed to handle the stress applied during the test. These findings are supported by Salahuddin et al. (20) who find that in certain circumstances involving mental and physical stress, SNS is activated while PNS will be at low and in turn causes an increase in heart rate. The participants of group B failed to control emotions and stress during the test and subsequently demonstrated low level of concentration than the participants of group A.

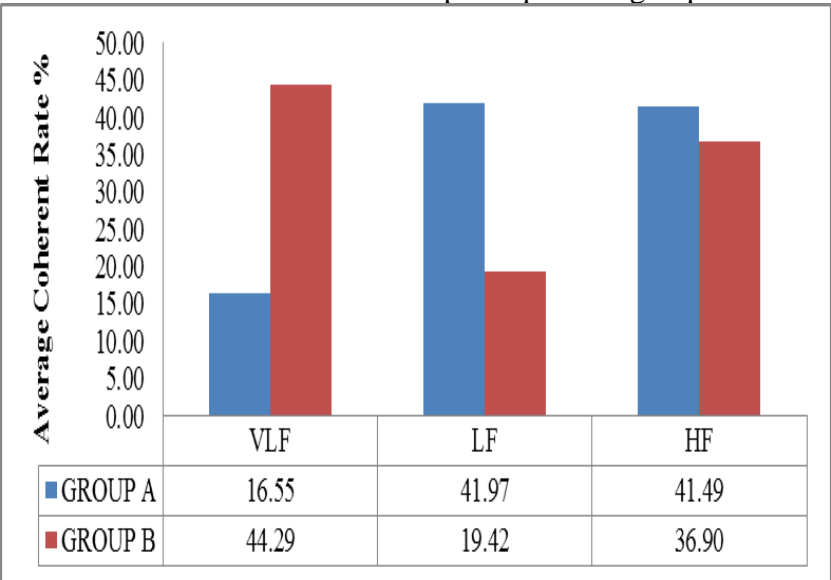

Fig. 4: Average Coherent Rate of HRV Power Spectrum in Stroop Test

Other than based on the Average Coherent Rate recorded via Multimedia Biofeedback Software, the instructors also recorded the data of error made by the participants during the test. Figure 5 shows the average of time and error made by the participants in the Stroop test. On average, participants of group A completed the first section within 15 seconds with an average error of 2 , while for section 2, the participants completed it with an average duration of 60 seconds and 5 errors. The participants of group B on the other hand, took longer period of time to complete; 30 seconds for section 1 and 80 seconds for section 2 . While in terms of errors made, it shows higher results than group A; 8 errors for section 1 and 12 errors for section 2 .

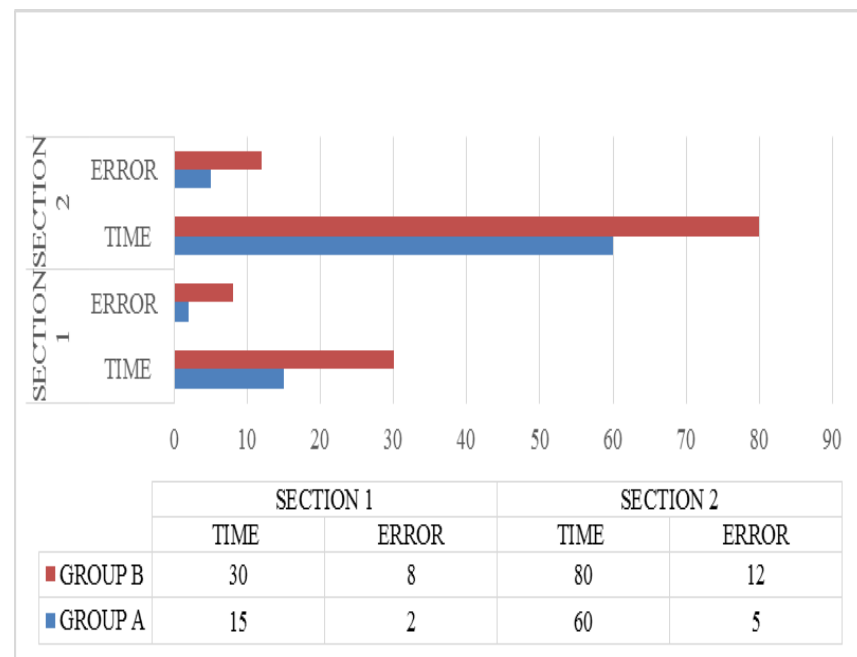

Fig. 5: Average Time and Errors in the Stroop Test Conducted on the Participants of Group A and B

Based on the findings of this study, it shows that both analyses indicate that there is consistency based on the HRV power spectrum and duration of the test and the error made by the participants. Through Stroop Test, the value of discipline in the aspect of concentration and regularity can be assessed. According to Hamalik (21), the ability to think (cognitive flexibility) is one of the characteristics of discipline because through this feature individuals who are able to think positively will be shaped and subsequently selfregulation will be more organised.

\section{Conclusion}

Overall, the study finds that the Stroop Test is one of the tests that can be used to assess the level of individual discipline. Apart from that, ongoing trials can increase the level of individual concentration and subsequently will be able to increase the level of discipline. This test is also able to introduce a new dimension in shaping individual behaviour that is more positive and will reduce social problems within the community as well as will increase the value of individual productivity. In addition, the increase in individual discipline will be able to raise the performance level and in turn will have an impact on the aspect of identity. Additionally, for individuals who have a low score should be given a therapist to increase their level of discipline as they are the driving force of the nation's future. In addition, when disciplinary problems among students can be overcome, social problems can be reduced.

\section{References}

[1] Foucault M. Security, territory, population: lectures at the Collège de France, 1977-78: Springer; 2007.

[2] Cawood J. Student Leadership in Schools: benevolent or malevolent. Gifted Education International. 1992;8(1):32-5.

[3] Andini P, Herrhyanto N, Suherman M. APLIKASI MULTIVARIATE GEOGRAPHICALLY WEIGHTED REGRESSION MENGGUNAKAN SOFTWARE MATLAB. Jurnal EurekaMatika. 2018;5(1):98-105.

[4] Robertson D, Low PA, Polinsky RJ. Primer on the autonomic nervous system: Academic Press; 2011.

[5] Ellenby MS, McNames J, Lai S, McDonald BA, Krieger D, Sclabassi RJ, et al. Uncoupling and recoupling of autonomic regulation of the heart beat in pediatric septic shock. SHOCKAUGUSTA-. 2001;16(4):274-7.

[6] Akselrod S, Gordon D, Ubel FA, Shannon DC, Berger A, Cohen RJ. Power spectrum analysis of heart rate fluctuation: a quantitative probe of beat-to-beat cardiovascular control. science. 1981;213(4504):220-2.

[7] McCraty R, Atkinson M, Tiller WA, Rein G, Watkins AD. The effects of emotions on short-term power spectrum analysis of heart rate variability. The American journal of cardiology. 1995;76(14):1089-93. 
[8] Wijsman J, Grundlehner B, Liu H, Hermens H, Penders J, editors Towards mental stress detection using wearable physiological sensors. Engineering in Medicine and Biology Society, EMBC, 2011 Annual International Conference of the IEEE; 2011: IEEE.

[9] Copie X, Hnatkova K, Staunton A, Fei L, Camm AJ, Malik M. Predictive power of increased heart rate versus depressed left ventricular ejection fraction and heart rate variability for risk stratification after myocardial infarction: results of a two-year follow-up study. Journal of the American College of Cardiology. 1996;27(2):270-6.

[10] Kamath MV, Fallen EL. Power spectral analysis of heart rate variability: a noninvasive signature of cardiac autonomic function. Critical reviews in biomedical engineering. 1993;21(3):245-311

[11] Dekker JM, Schouten EG, Klootwijk P, Pool J, Swenne CA, Kromhout D. Heart rate variability from short electrocardiographic recordings predicts mortality from all causes in middle-aged and elderly men: the Zutphen Study. American journal of epidemiology. 1997; 145(10):899-908.

[12] Lohr I. The influence of effort on impairments of attention associated with major affective disorders: Clark University; 1995.

[13] Imbrosciano A, Berlach RG. The Stroop test and its relationship to academic performance and general behaviour of young students. Teacher Development. 2005;9(1):131-44.

[14] Webster JG. Design of pulse oximeters: CRC Press; 1997.

[15] Haahr MT, Hróbjartsson A. Who is blinded in randomized clinical trials? A study of 200 trials and a survey of authors. Clinical Trials. 2006;3(4):360-5

[16] Šiška E. The Stroop colour-word test in psychology and biomedicine. Acta Universitatis Palackianae Olomucensis Gymnica. 2002;32(1).

[17] Cahill L, Alkire MT. Epinephrine enhancement of human memory consolidation: interaction with arousal at encoding. Neurobiology of learning and memory. 2003;79(2):194-8

[18] Shah AJ, Su S, Veledar E, Bremner JD, Goldstein FC, Lampert R, et al. Is heart rate variability related to memory performance in middle aged men? Psychosomatic medicine. 2011;73(6):475.

[19] Frewen J, Finucane C, Savva GM, Boyle G, Coen RF, Kenny RA. Cognitive function is associated with impaired heart rate variability in ageing adults: the Irish longitudinal study on ageing wave one results. Clinical Autonomic Research. 2013;23(6):313-23.

[20] Salahuddin L, Cho J, Jeong MG, Kim D, editors. Ultra short term analysis of heart rate variability for monitoring mental stress in mobile settings. Engineering in Medicine and Biology Society, 2007 EMBS 2007 29th Annual International Conference of the IEEE; 2007: IEEE.

[21] Hamalik O. Pendidikan guru: berdasarkan pendekatan kompetensi: Bumi Aksara; 2002. 